\title{
El sector público en los modelos de crecimiento y desarrollo económicos: una aproximación al caso de la economía mexicana
}

\author{
The public sector models of economic growth and development: an aproach \\ to the case of the mexican economy
}

\begin{abstract}
Resumen
El artículo aborda el tema de los factores que inciden en el crecimiento de la economía mexicana y uno de ellos fue el abierto intervensionismo estatal que se desplegó a través de sus múltiples instituciones. Sin embargo, a raíz del proceso de apertura los resultados en términos de crecimiento y desarrollo para la economía y sociedad mexicanas no han sido satisfactorios, por lo que urge recobrar la senda del crecimiento sostenido. En este escenario y a la luz de la histórica trascendencia de las instituciones gubernamentales es que en el presente trabajo se recurre a la estimación econométrica de una función de producción agregada que considera al capital, al trabajo, al avance tecnológico y al gobierno y sus instituciones consideradas a través del gasto público, como los principales elementos que en los últimos lustros han determinado el crecimiento de la economía mexicana y que lo pueden potencializar en el futuro cercano.
\end{abstract}

\footnotetext{
Palabras claves

- Crecimiento económico

- intervencionismo estatal

- instituciones

- apertura económica ajuste macroeconómico

- función de producción

- modelos econométricos
}

\begin{abstract}
The article discusses the factors that influence the growth of the Mexican economy and one of them was the open interventionism of the State that unfolded through its various institutions. However, since the process of opening of the economy, the results in terms of growth and development for the Mexican economy and society have not been satisfactory, therefore it is urgent to recover the path of sustained growth. In this scenario and due to the historical trascendence of government institutions, this paper turns to an econometric estimation of an aggregate production function that considers the capital, labor, technological progress and the government and its institutions considered through public expenditure, as the main elements that in the last decades have led the growth of the Mexican economy and that can potentiate the near future.
\end{abstract}

JEL: HI I, H53, OI 2

\section{Introducción'}

El presente artículo aborda el tema de los factores que inciden en el crecimiento de la economía mexicana en presencia del Estado, el cual en la actual estrategia de apertura económica se ha visto minimizado en virtud de considerarlo poco funcional a la misma. Sin embargo los resultados en términos de crecimiento y desarrollo para la economía y sociedad mexicana, distan mucho de lo que se había prometido que sucedería en caso de adoptarla; en este sentido es que se hace necesario conocer el verdadero papel del gobierno mexicano y de sus instituciones en términos de su

I Este trabajo es un avance de investigación del proyecto Modelos de sustitución de importaciones y de apertura una comparación de variables macroeconómicas y empresariales, que concentra el trabajo de varios de los integrantes de la Unidad de Investigación en Economía Aplicada del IIEC-UNAM. 
real aporte al crecimiento económico y ese es el sentido del presente trabajo que, recurriendo a la teoría económica existente detrás de las funciones de producción agregada, propone un sencillo modelo que considera al capital, al trabajo, al avance tecnológico y sobre todo al gobierno considerado a través del gasto público, como los principales elementos que determinan el crecimiento de la economía mexicana.

El presente trabajo se divide para su tratamiento en seis partes la primera introductoria en donde se esboza el carácter general de la investigación; la segunda se dedica al análisis de la globalización y su impacto en las naciones emergentes como es el caso de la economía mexicana; en la tercera se discuten las corrientes que teorizan alrededor de los determinantes del crecimiento económico; en la cuarta se analiza al modelo sustitutivo de importaciones cambiado a raíz de la crisis de deuda externa, por una estrategia que para crecer proponía el repliegue del Estado por medio de la privatización, desregulación y apertura unilateral de la economía mexicana a los flujos comerciales y financieros internacionales que desembocó en la primer crisis del modelo de apertura iniciada a raíz del error de diciembre de 1994 que implicó caídas subsecuentes de la economía mexicana, sin que se alcanzaran las tasas de crecimiento prometidas por dicha estrategia; la quinta parte analiza los elementos que inciden en el crecimiento económico de México, mediante una función de producción que se valida recurriendo a la econometría estructural; finalmente en la sexta y última parte, se presentan las conclusiones y recomendaciones de política económica en materia de crecimiento económico.

\section{Globalización}

El fenómeno de la globalización puede entenderse como un proceso esencialmente económico que actualmente se vive a escala planetaria en prácticamente la mayoría de los países en algunos viviéndose con mayor o menor nivel de intensidad, por lo que puede definirse desde diversas perspectivas y una de ellas es la del economista mexicano J.Ayala quien opina que se trata de:

La globalización no es solamente ni aun primariamente un fenómeno económico. Tampoco es la simple extensión y conexión de las economías en el mundo a través de las inversiones y el intercambio comercial, lo cual ha ocurrido en mayor o menor grado a lo largo de muchos años. Por el contrario, la globalización es un nuevo proceso que transforma radicalmente las nociones convencionales del tiempo y el espacio del desarrollo económico tal como se conocían en los años cincuenta. Esta transformación es propiciada por la revolución de las comunicaciones, los transportes y la teleinformática, lo cual ha permitido acceder a grandes bancos de información, a la difusión acelerada de esta, pero también a su control por unos cuantos gobiernos y corporaciones. La globalización es también la entrada a lo que Sakaiya denomina la sociedad del conocimiento basado en la producción de conocimientos" (Ayala, 2002). 
Existe otra opinión que recupera lo esgrimido en el Informe Sobre Desarrollo Humano de 1999 del Programa de las Naciones Unidas para el Desarrollo (PNUD), donde se afirma que:

\begin{abstract}
Hay que reconocer como un factor de esperanza que la globalización del inicio del siglo XXI, al permitir una comunicación rápida y directa, no sólo entre los gobiernos sino entre los miembros de la sociedad civil, rebasa los movimientos de mercancías, capitales y tecnología y presenta una enorme potencialidad para erradicar la pobreza mundial y obtener un progreso generalizado, siempre y cuando existan los compromisos y mecanismos para construir una comunidad internacional no sólo interdependiente, sino incluyente, con valores compartidos en derechos humanos, sustentabilidad del planeta y democracia como forma de gobierno y de convivencia social. "Mundialización con ética, equidad, inclusión, seguridad humana, sostenibilidad ecológica y desarrollo” (Martínez, 200I).
\end{abstract}

Sin embargo se debe condicionar a la globalización para que cumpla con los objetivos del proyecto nacional de cada país con objeto de que sirva al desarrollo, aprovechando la bonaza económica de las economías desarrolladas que pueden invertir sus excedentes para acelerar el desarrollo de los países atrasados; en este sentido, el único ente económico-social que puede hacerlo es el Estado ya que dispone de múltiples instrumentos gubernamentales de intervención, que son armonizados bajo el concepto de políticas públicas y que de hecho se hace por parte de los países desarrollados (OCDE) en este mismo contexto globalizador (Martínez, 200 I).

\title{
3. Elementos teórico-conceptuales sobre la intervención del estado en la economía.
}

La intervención del Estado en la economía es un tema que ha estado presente en los debates más importantes de la ciencia económica en tal grado que A. Smith dedica, aun siendo escéptico de la intervención estatal en la economía, varios capítulos de la Riqueza de las Naciones a este problema subdividiéndolo para su tratamiento en los temas de ingreso, gasto y deuda pública que son abordados en el capítulo I partes I, 2 y 3 del LibroV (Smith, 2009). Los referentes teóricos que utilizó fueron las opiniones de economistas que lo antecedieron que eran de orientación mercantilistafisiócrata pero que motivó a sus seguidores para profundizar en ella; en este sentido las corrientes de pensamiento económico que se identifican con ella son dos:

- los que se mantienen fieles a la tradición iniciada por Smith, en el sentido de mantener al mínimo nivel posible el proceso de intervención del Estado en la economía, y de la cual son seguidores autores clásicos como Say, Ricardo, y Mill, economistas neoclásicos como Walras, Menger y Jevons, así como escuelas más 
contemporáneas como la de expectativas adaptativas, racionales de Sargent y Barro, y finalmente la macroeconomía del equilibrio de Lucas.

- Existe otra corriente que se manifiesta a favor de procesos de intervención estatal mas activos en la economía, entre estas opiniones se encuentran economistas clásicos como Malthus, Bentham, Lizt y Marx, seguidos de enfoques más contemporáneos pero coincidentes con esta última línea interpretativa de argumentación. Entre las opiniones más importantes destacan la escuela de Cambridge con Marshall, Pigou y Pareto que inauguraron el estudio de la economía del bienestar bajo un enfoque esencialmente microeconómico; la revolución Keynesiana de carácter macroeconómico y sus enfoques posteriores entre los que destacan: la escuela poskeynesiana con autores como Srafa, Kalecky, Robinson y Samuelson; la neokeynesiana dinamizadora de Harrod y Domar; de microfundamentación de Tobin y Baumol; la síntesis neoclásica de Hicks, Hansen y Patinkin; la de los nuevos keynesianos de Stiglitz, Mankiew y Romer; y finalmente la Teoría del Desarrollo y su enfoque latinoamericano cepalino, que ubican al gobierno y sus instituciones como elementos claves a considerar en el crecimiento y desarrollo económicos.

\section{I El Estado y sus instituciones en los modelos de crecimiento y desarrollo económicos}

Explicar el desarrollo económico implica tomar en cuenta factores de tipo cuantitativo y cualitativo por lo que no es suficiente considerar únicamente al crecimiento, ya que dicho factor representa una condición necesaria pero no suficiente para que la economía se desarrolle a plenitud, como Blanchard al establecer las diferencias entre la Teoría del Desarrollo Económico y la Teoría del Crecimiento Económico que considera al papel de la tecnología en sentido estricto, en relación con el fascinante reino de la Economía del Desarrollo que incorpora al conjunto de instituciones de un país en una definición de tecnología en sentido amplio (Blanchard, 2000).

La afirmación anterior representa la punta de iceberg de una prolífica línea de investigación iniciada a fines de las décadas de los años treinta y cuarenta del siglo XX, con los modelos de crecimiento keynesianos escritos por Harrod y Domar ( $\mathrm{Ha}-$ rrod, 1939; Domar, 1947) profundizados por Solow en sus modelos de Contabilidad de Crecimiento (Solow, 1956; Solow, 1957) que explican al dicho proceso a partir de la inversión realizada en los factores productivos trabajo (educación), capital (tasa de ahorro) y tierra (recursos naturales).

La consideración explícita del avance tecnológico en los modelos convencionales de crecimiento exógeno y su refutación empírica han llevado a economistas de 
formación neoclásica como Romer y Lucas a proponer modelos de crecimiento endógeno que explicitan al factor tecnológico (Romer, I986; Lucas, 1988), situación que los ha llevado a recuperar aportes previos de economistas como Schumpeter que define el proceso de cambio estructural como el crecimiento económico a partir de la destrucción creativa (Schumpeter, 1934), y el modelo de acumulación del conocimiento a partir de la experiencia adquirida al participar en los procesos productivos (Arrow, 1962).

La consideración del cambio tecnológico derivó en los últimos años en los actuales modelos de crecimiento endógeno, del tipo:

$$
Y=F(K, L, N, H, A)
$$

Donde:

Y representa a la producción

K al capital

$\mathrm{L}$ a la fuerza de trabajo

$\mathrm{N}$ a la tierra

$\mathrm{H}$ al capital humano

A representa a la tecnología (como residuo de Solow)

Los modelos de crecimiento endógeno se han complementado con aportes propuestos por economistas institucionalistas que han ampliado a la función de producción, al considerar explícitamente el papel que juegan en el crecimiento y el desarrollo los factores institucionales $(\mathrm{Qn})$; entre estos factores se encuentra la protección de los derechos de propiedad; el respeto a los contratos; la cantidad de trámites burocráticos requeridos para llevar a cabo la producción; la existencia de tratados comerciales, entre otros.

Con base en los elementos anteriormente considerados la función de producción en su versión ampliada quedó finalmente establecida de la siguiente manera:

$$
Y=F(K, N, L, H, A, Q n)
$$

La función de producción tiene alternativamente una versión simplificada que se expresas de la siguiente forma (Rodríguez, 2009: 42): 


$$
Y=F(K, L, A, Q n)
$$

Los modelos de crecimiento endógeno incorporan al proceso tecnológico en su explicación analítica de los determinantes del crecimiento, pero lo siguen considerando en sentido estricto mientras que otras corrientes lo conciben en sentido amplio destacando entre las más importantes las siguientes:

- La institucionalista (Polanyi, 2002);

- La neoinstitucionalista (North, 1990);

- La evolucionista neoschumpeteriana (Hanush; Pyka, 2007)

- El enfoque del Desarrollo Endógeno (Vázquez, 2002).

-

Las teorías mencionadas sostienen que el cambio tecnológico es determinado por el conjunto de instituciones formales (leyes-reglamentos) e informales (libertad e igualdad) que junto con otros elementos explican el desarrollo económico exitoso de los países avanzados y el de los emergentes; en este sentido el cambio tecnológico inducido por el entorno institucional delineado por el Estado, ha implicado su análisis en dos vertientes complementarias (Bandeira, 2009):

- la perspectiva de los sistemas políticos (democracia-transparencia vs. dictadura-opacidad).

- la perspectiva de la administración pública que utiliza como instrumento a las políticas públicas para identificar los problemas, evaluarlos, confrontar las posibles opciones de resolución y proyectar sus resultados (Bardach, 2004).

El análisis institucional desde la perspectiva de los sistemas políticos ha recibido menor atención de analistas, investigadores y decisores que se explica por la complejidad multifactorial en que dicho factor se desenvuelve, pero en cambio este análisis si se realizó en el campo de la Administración Pública por el impulso de la Nueva Gestión Pública que refuncionaliza al modelo weberiano convencional incorporando criterios de mercado que ha sido tomado como guía de las reformas institucionales implementadas en países de diverso nivel de desarrollo (Bandeira, 2009); en este sentido y en el caso de economías de bajo desempeño económico como la mexicana, dicho proceso no arrojó los resultados esperados en términos de crecimiento debido a que dicha estrategia no fue calibrada al momento de su implementación por parte de la vertiente política. 


\section{El sector público en el desarrollo y apertura de la economia mexicana}

Un elemento que caracterizó al modelo sustitutivo de importaciones que México utilizó como estrategia de crecimiento económico para alcanzar su desarrollo, fue la presencia activa del Estado en la economía que formó con el tiempo la llamada economía mixta mexicana permitiéndolo avanzar en materia de industrialización y distribución del ingreso que pudo alcanzarse gracias a los programas de inversión pública instrumentados por la Comisión de Inversiones dependiente de la Secretaria de la Presidencia y por otras secretarías de Estado, que en conjunto tuvieron la encomienda de afrontar los problemas derivados de las imperfecciones de mercado, propios de una nación en vías de desarrollo, que al ser resueltos mejoraron su perfil económico como país y le aseguró a la sociedad mexicana el acceso a legítimos niveles de bienestar e igualdad.

La serie de acontecimientos y desequilibrios internos y externos que se presentarse en la economía mexicana a principios de los años ochentas propiciaron el abandono de la estrategia de intervencionista estatal, para dar paso a un profundo proceso de reformas económicas conocidas como la estrategia de ajuste y cambio estructural en donde privatización, desregulación y apertura económica fueron los mecanismos mediante los cuales se superarían los desequilibrios macroeconómicos gestados en la etapa inmediata anterior que se concentraban en controlar el problema inflacionario como un prerrequisito necesario para regresar a la senda del crecimiento económico con estabilidad.

La adopción de medidas neoliberales en un ambiente crecientemente globalizado implicó para la economía mexicana el desmantelamiento de su economía mixta que se hizo bajo el argumento de que el Estado y sus instituciones resultan ineficientes desde el punto de vista productivo, además de ser considerados dispendiosos por lo que su participación económica debía de llevarse al mínimo y ser sustituida por agentes privados; esto último comenzó a llevarse de manera efectiva a partir de 1983, que es cuando se iniciaron los procesos económicos impulsores de las medidas de política económica adoptadas para salir de la crisis de la deuda externa de 1982 o las tomadas para superar las consecuencias derivadas del error de diciembre de 1994. 


\section{I Ajuste macroeconómico, cambio estructural y crisis financiera de la economía mexicana}

El primer bloque de medidas de política económica que se empelaron en México para afrontar a la crisis de la deuda externa de 1982 se caracterizó por la adopción de un programa de estabilización macroeconómica y cambio estructural (Rogosinsky, 1997), que se implementó entre los años de 1983 a 1994 y donde se comenzó a desmantelar aceleradamente a la industria paraestatal mexicana mediante el cierre, extinción y privatización de empresas paraestatales, pasando de I,I55 en 1982 a 269 en el año de 1994 que es la fecha en que concluye esta primera etapa (Manrique; Escamilla, 1996); esta etapa se implementó con el apoyo de importantes cambios constitucionales, como fueron las reformas del artículo 25 en donde el Estado Mexicano se erige como rector de la economía y el 28 donde el Banco de México asume como su principal función el control de precios dejando de lado el estímulo al crecimiento económico.

El segundo bloque de medidas de política económica iniciaron con el ajuste macroeconómico adoptado a partir de 1995 para superar la crisis económica generada por el error de diciembre de 1994 y consistieron en devaluación del tipo de cambio, incremento de impuestos, recortes al gasto público y restricción monetaria; en este sentido, la adopción del programa de ajuste macroeconómico implicó la quiebra del sector bancario y el restablecimiento del equilibrio macroeconómico que se perdió debido a la irrestricta apertura comercial y financiera de la economía mexicana (Ros; Moreno, 2010).

4.2 Evolución de los factores productivos de la economía mexicana en la era de la apertura y la globalización.

El Fondo Monetario Internacional en su Ranking para el año de 20 I I que mide a las principales economías del mundo sitúa a la mexicana en el lugar I4 con un PIB de I,004,042 mdd; este nivel es inferior al que registraron las economías de países desarrollados como EU, Japón, Alemania, Francia, Inglaterra o al que registran países emergentes como Brasil, Rusia, India, China y Sudáfrica (BRICS ), pero es superior al de países como Corea del Sur, Holanda, Turquía e Indonesia, como puede observarse en el cuadro I. 


\section{Cuadro I}

Producto interno bruto de las principales 20 Economías del mundo

(Millones de dólares estadounidenses)

\begin{tabular}{|c|c|c|}
\hline 1 & Estados Unidos & 14624184 \\
\hline 2 & China & $5745 \quad 133$ \\
\hline 3 & Japón & 5390897 \\
\hline 4 & Alemania & 3305898 \\
\hline 5 & Francia & 2555439 \\
\hline 6 & Reino Unido & 2258565 \\
\hline 7 & Italia & 2036687 \\
\hline 8 & Brasil & 2023528 \\
\hline 9 & Canadá & I 563664 \\
\hline 10 & Rusia & 1476912 \\
\hline II & India & I 430020 \\
\hline 12 & España & I 374779 \\
\hline 13 & Australia & 1219722 \\
\hline 14 & México & 1004042 \\
\hline 15 & Corea del Sur & 986256 \\
\hline 16 & Países Bajos & 770312 \\
\hline 17 & Turquía & 729051 \\
\hline 18 & Indonesia & 695059 \\
\hline 19 & Suiza & 522435 \\
\hline 20 & Bélgica & $46 \mid 331$ \\
\hline
\end{tabular}

Fuente: cuadro tomado del Ranking de Economías Mundiales 20 I I, publicado por el FMI.

Entre las causas mas importantes que explican la posición de la economía mexicana en el ranking internacional, se encuentra el comportamiento de sus principales factores de la producción el capital y el trabajo que reportan crecimientos moderados y permanentes entre los años de 1980 y 2008; en este sentido, en lo que respecta al factor capital su comportamiento durante el período fue errático y evidente en los años previos a la crisis de 1994, recuperándose a partir del año de 1996 y manteniéndose en dicha condición hasta el momento, situación que contrasta con el comportamiento registrado por el factor trabajo que durante el período crece uniformemente, como se puede observarse en el cuadro 4. 


\section{Cuadro 2}

Evolución del trabajo y del acervo de capital en México (1980-2008)

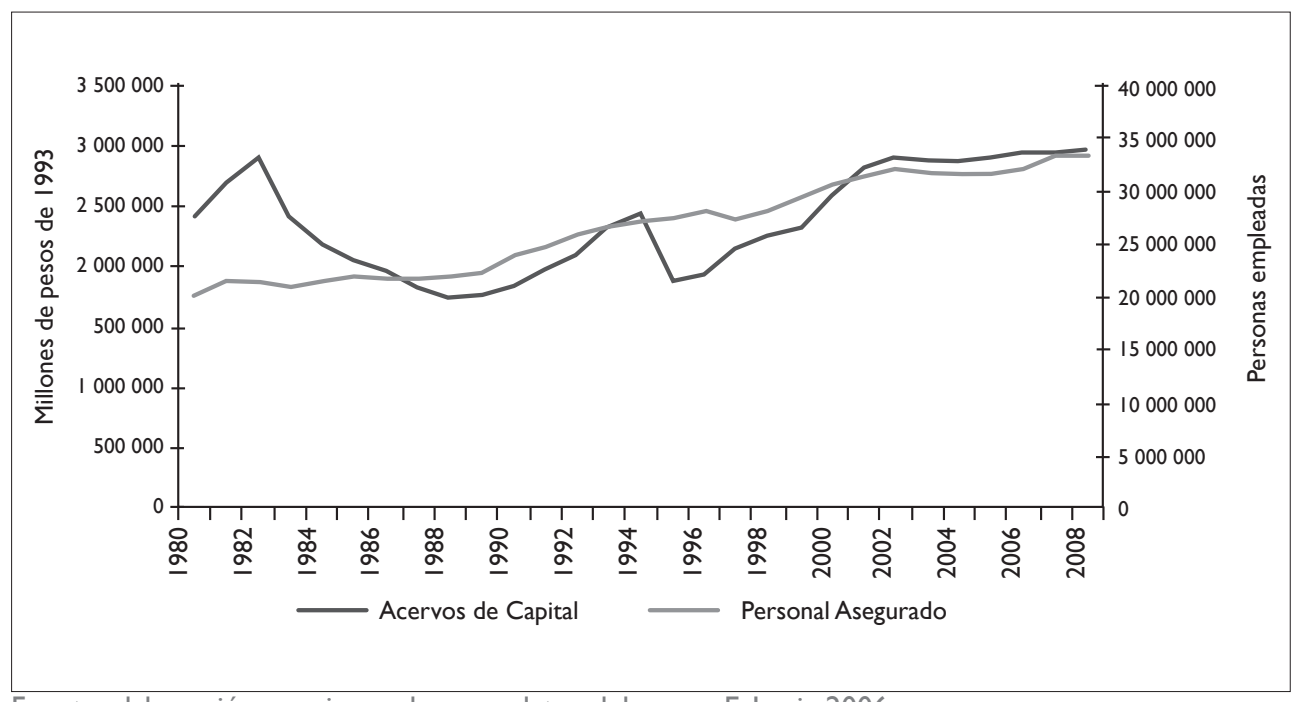

Fuente: elaboración propia con base en datos del INEGI y E. Loria 2006.

Con base en lo anterior puede afirmarse que el comportamiento ascendente registrado durante el periodo por los factores de la producción trabajo y capital no fue suficiente para sacar a la economía mexicana del subdesarrollo en que actualmente se encuentra, siendo motivo de preocupación para los analistas y hacedores de la política económica que no alcanzan a explicarse, porque después de tantos años de reformas en la economía mexicana esta no crece de la forma en que en ese mismo tiempo lo han hecho las economías emergentes.

Un elemento clave que incide en el crecimiento de las economías es todo lo relacionado con el gasto público (corriente y de inversión) que en México en los últimos años acusa una trayectoria de bajo perfil; esto último se comprueba, al observar el comportamiento en términos reales de las variables formación bruta de capital fijo gubernamental y el gasto corriente integrado por las erogaciones gubernamentales destinadas a educación y salud que en el período comprendido entre 1982 y 1995 se estancan y se recuperan solo a partir del año de 1996. Sin embargo, dicha recuperación ha sido muy débil y ha contribuido muy poco al crecimiento de la economía mexicana en su conjunto no ha pasado del $2 \%$ promedio anual resultando inferior al $6.5 \%$ que por más de cincuenta años registró, como puede observarse en el cuadro 3. 


\section{Cuadro 3}

Evolución del PIB, del gasto público en salud, educación e inversión pública

1980-2008 (Escala logarítmica)

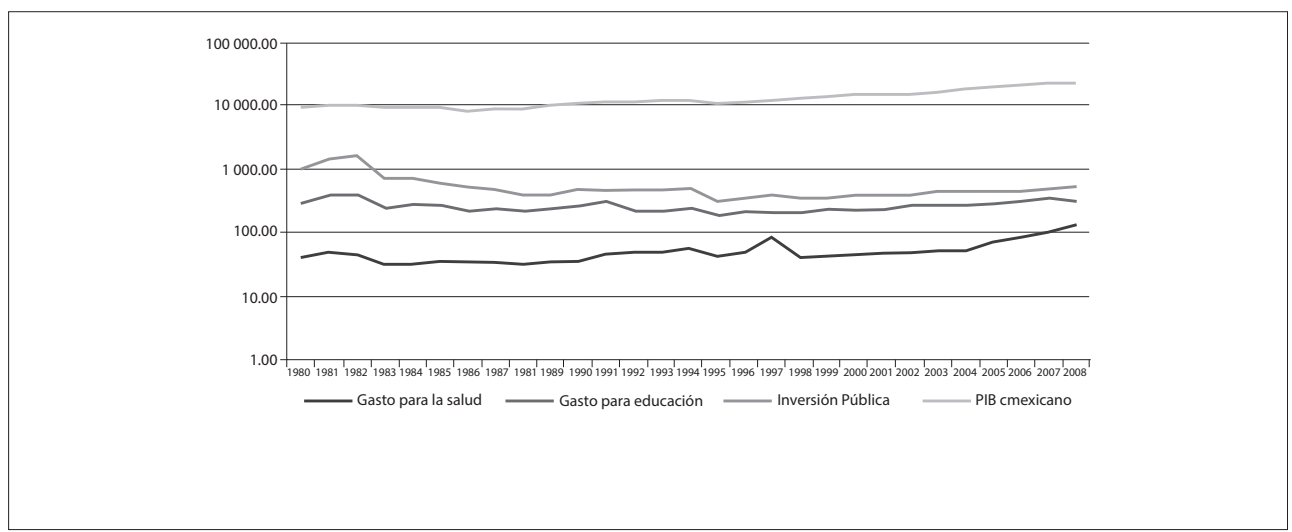

Fuente: elaboración propia con base en datos del INEGI.

El comportamiento del gasto público mexicano no fue uniforme durante esta etapa porque el gasto público en inversión se contrajo sistemáticamente sobre todo a partir del año de 1983, sin que haya podido recuperar los niveles que tenía hasta antes de la crisis de la deuda externa cuando superar el $40 \%$ del PIB; en este sentido, el gasto público en educación cae a partir de 1983 y se mantiene en ese nivel hasta 1995, comenzando a crecer a partir de 1996, como se observa en el cuadro 4.

\section{Cuadro 4}

Gasto público, educación y formación bruta de capital fijo (1980-2008)

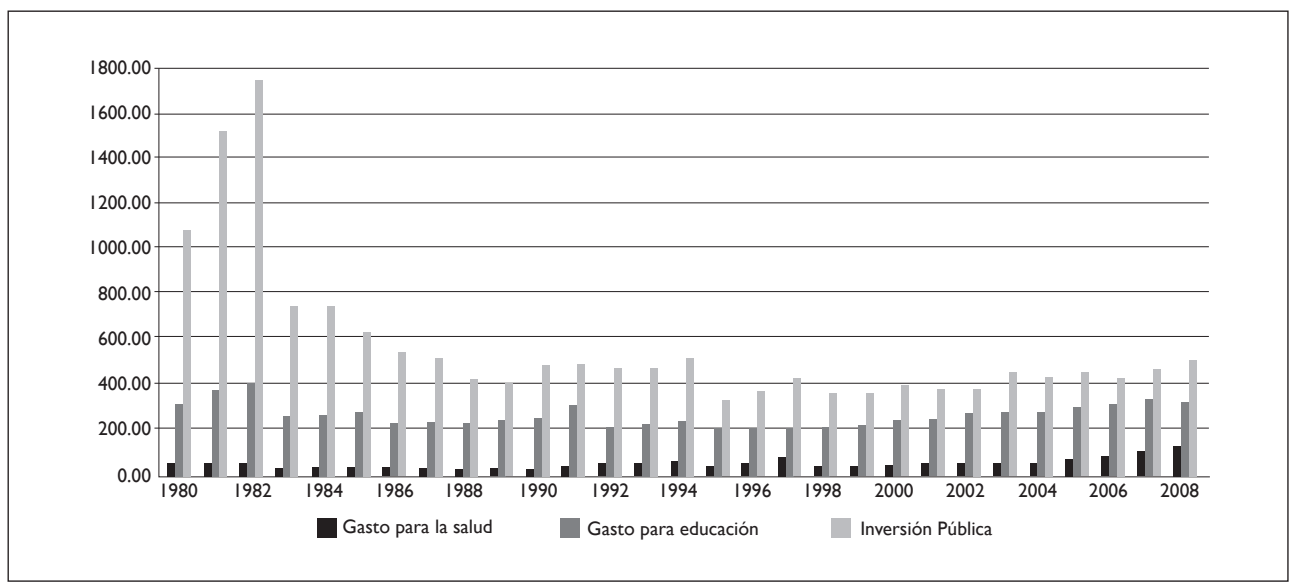

Fuente: elaboración propia con base en datos del INEGI. 
Lo sucedido con el gasto público en educación se repite en otros rubros importantes de gasto social sobre todo en aquellos que tienen que ver con la salud y combate a la pobreza, lo que permite afirmar que durante este último periodo el gasto relacionado con el desarrollo social se contrajo y dicha caída contribuyó a configurar el cuadro recesivo que caracteriza al conjunto de la economía mexicana en los últimos años.

Finalmente si se aspira a recuperar las tasas de crecimiento que por más de 50 años fueron del 6.5 promedio anual, lo primero que debe hacerse desde el punto de vista de la política económica es restablecer los niveles de gasto público que se tenían hasta antes de la crisis económica de la deuda externa mexicana de 1982. Asimismo se requiere determinar empíricamente la importancia, en términos del crecimiento de la economía mexicana, que tienen las principales variables del gasto público objetivo que se abordará en el siguiente epígrafe.

\section{Modelos de crecimiento para la economía mexicana en presencia de instituciones públicas}

Es un hecho incontrovertible que las instituciones en general y las gubernamentales en particular contribuyen al crecimiento y desarrollo de las economía, así lo comprueban diversos estudios hechos al respecto. Entre los mas importantes destaca el realizado por Acemoglu en donde se analiza el nivel de protección contra una expropiación en países de alto, medio y bajo nivel de desarrollo económico; en este sentido en el contexto de los resultados del estudio realizado para múltiples países, la economía mexicana se ubica en una posición media alta indicativa de que a mayor PIB existe mayor probabilidad de que se tenga una mejor en la protección de los derechos de propiedad contra una expropiación arbitraria por parte de las autoridades, por ejemplo. (Robbins, 2004)

Existen otros estudios de corte internacional que analizan a las economías de México y Latinoamérica en torno a los factores productivos e instituciones públicas que inciden en su crecimiento; entre los más importantes destacan los estudios de Campos y Jeffrey (Campos; Jeffrey, 1998), el de Barro (Barro, I99I), el de la Oficina del Congreso de EU (OFC, 1993), y el de Levine (Levine, 1993). También hay estudios hechos en México bajo la misma temática destacando los realizados por Díaz y Sáenz, Díaz, Loría y finalmente el de Estrada que en unión con los estudios de corte internacional han permitido el avance en el conocimiento de los factores que determinan el crecimiento y el desarrollo de la economía mexicana.

El trabajo pretende avanzar en el estudio del impacto que tienen en el crecimiento económico factores como: el trabajo considerado a través del número de 
empleados laborando formalmente en una empresa; el capital que es captado a través de la formación bruta de capital fijo de las empresas (FBKF) y factores de tipo cualitativo como los de tipo cualitativo, entre los que se encuentran las instituciones públicas consideradas específicamente a través del gasto público que se ejerce por la vía , en el crecimiento económico de México en el periodo que va de 1980 a 2008; en este sentido es que se propone a la función de producción tipo Cobb-Douglas en donde los factores cuantitativos como el gasto público son incluidos explícitamente en una variable $(G)$, mientras que factores de tipo cualitativo $(A)$ son considerados a través de incorporar al considerar las instituciones gubernamentales y la tecnología están contempladas en el famoso residuo de Solow, de tal manera que con base en la función de producción tipo Cobb-Douglas, originalmente propuesta en 1899 por Wicksell, propongo la siguiente especificación con tres insumos, esto es, capital, trabajo, gasto gubernamental y el residuo de Solow, en donde la producción en cualquier punto $t$ viene dada por: ${ }^{2}$

$$
Y_{(t)}=A_{(t)} K_{(t)}^{\alpha} L_{(t)}^{\beta} G_{(t)}^{c}
$$

En donde:
A representa a la constante
$\mathrm{K}$ representa al factor capital
$L$ representa al factor trabajo
$G$ representa al gasto público
En donde los superíndices $\alpha, \beta$, ç $>0$ y $\alpha+\beta+c ̧=$ I

La función de producción puede especificarse en forma logarítmica, que dando estipulada de la siguiente manera:

2 Donde la variable Y representa la producción de la economía mexicana medida por el PIB, la variable $\mathrm{K}$ representa a los acervos de capital de la economía mexicana que se obtuvieron con el método de inventarios perpetuos ajustados (MIPA) con base en la formación bruta de capital fijo público y privado en México, y $\mathrm{G}$ es una variable que desagregada representa al gasto público social (salud, educación, alimentación, vivienda) y puede también incluir el gasto en infraestructura. Por su parte $\alpha$ representa la elasticidad parcial de la producción respecto al capital, $\beta$ es la elasticidad parcial de la producción respecto al trabajo, y ç es la elasticidad parcial de la producción respecto a la variable que representa al gasto público. 


$$
\operatorname{Ln}\left(\mathrm{Y}_{\mathrm{t}}\right)=\mathrm{a}_{\mathrm{t}}+\operatorname{Ln} \alpha \mathrm{K}_{\mathrm{t}}+\operatorname{Ln} \beta \mathrm{L}_{\mathrm{t}}+\operatorname{Ln} \mathrm{çG}_{\mathrm{t}}
$$

En donde:

$$
\begin{aligned}
& a=\operatorname{Ln}(\mathrm{A}) \text { que es el término constante } \\
& \alpha=\text { representa la elasticidad parcial de la producción respecto al capital (signo } \\
& \text { positivo) } \\
& \beta=\text { representa la elasticidad parcial de la producción respecto trabajo (signo } \\
& \text { positivo) } \\
& c ̧=\text { representa la elasticidad parcial de la producción respecto gasto público }
\end{aligned}
$$

La información estadística disponible para la economía mexicana de las variables escogidas que corren del año de 1980 al de 2008 permitió estimar mediante la econometría estructural a la función de producción propuesta; en este ejercicio para captar la presencia de las instituciones gubernamentales, lo que se hizo fue tomar en cuenta el gasto público, pero desagregándolo en gasto público federal en educación y salud públicas, además de que el gasto en infraestructura se consideró explícitamente a través de una variable próxi.

Los resultados econométricos se presentan en el cuadro 5 que contiene los estadísticos representativos del ajuste econométrico, que en este caso resultó ser de tipo doblelogarítmico que incorpora explícitamente a los factores trabajo, capital y las instituciones gubernamentales consideradas estas últimas, a través del presupuesto destinado para dichos fines las cuales están incluidas implícitamente en el gasto público que para fines de la presente investigación se desagregó en los rubros de gasto público educativo, de salud y en infraestructura. 


\section{Cuadro 5}

Dependent Variable: LNPIBREAL 1993

Method: Least Squares

Date: 08/05/II Time: 14:00

Sample: I 29

Included observations: 29

\begin{tabular}{|c|c|c|c|c|}
\hline Variable & Coefficient & Std. Error & t-Statistic & Prob. \\
\hline C & -79.43768 & 24.43955 & -3.250374 & 0.0035 \\
\hline LNK & $2 . \mid 86231$ & 0.991375 & 2.205251 & 0.0377 \\
\hline LNPERSASEG & 4.864107 & 1.659884 & 2.930390 & 0.0075 \\
\hline LNGEDUC93 & -1.911815 & 1.513167 & -1.263452 & 0.2191 \\
\hline LNGSALUD93 & 0.474759 & 0.669560 & 0.709061 & 0.4854 \\
\hline LNFBKFRGOB & -2.626185 & $\mathrm{I} .1005 \mathrm{II}$ & -2.386332 & 0.0256 \\
\hline R-squared & 0.957552 & \multicolumn{2}{|c|}{ Mean dependent var } & 13.57576 \\
\hline Adjusted R-squared & 0.948325 & \multicolumn{2}{|c|}{ S.D. dependent var } & 2.484443 \\
\hline S.E. of regression & 0.564769 & \multicolumn{2}{|c|}{ Akaike info criterion } & 1.877192 \\
\hline Sum squared resid & 7.336173 & \multicolumn{2}{|c|}{ Schwarz criterion } & 2.160080 \\
\hline Log likelihood & -21.21928 & \multicolumn{2}{|l|}{ F-statistic } & 103.7688 \\
\hline Durbin-Watson stat & 1.179182 & \multicolumn{2}{|l|}{ Prob(F-statistic) } & 0.000000 \\
\hline
\end{tabular}

en donde:

C es la constante que resulta altamente significativa pero con signo negativo LNK es el logaritmo de los acervos reales de capital en México LNPERASEG es el logaritmo del número de trabajadores asegurados permanentemente por alguna institución de salud pública que resultó positivo LNGEDUC93 es el logaritmo del gasto público real en educación LNGSALUD93 es el logaritmo del gasto público real en salud LNFBKFRGOB es el logaritmo del gasto público real en inversión gubernamental

Los resultados del ejercicio econométrico anterior se confirman al analizar de manera paralela al cuadro 6 que contiene a la matriz de correlación que confirma los nexos económicamente existentes entre los factores productivos: trabajo, capital, instituciones en su vinculación con relación al producto interno bruto y su crecimiento. 


\section{Cuadro 6}

Matriz de correlación

\begin{tabular}{|c|c|c|c|c|c|c|}
\hline & $\mathrm{C}$ & LNK & LNPERSASEG & LNGEDUC93 & LNGSALUD93 & LNFBKFRGOB \\
\hline C & 597.2918 & 0.291100 & -36.56910 & 23.84399 & 13.38334 & -22.15286 \\
\hline LNK & 0.291100 & 0.982824 & -0.698172 & -0.003620 & 0.029253 & -0.387304 \\
\hline LNPERSASEG & -36.56910 & -0.698172 & 2.755214 & -1.651916 & -0.839276 & 1.672150 \\
\hline LNGEDUC93 & 23.84399 & -0.003620 & -1.651916 & 2.289675 & 0.354099 & -1.384713 \\
\hline LNGSALUD93 & 13.38334 & 0.029253 & -0.839276 & 0.354099 & 0.448311 & -0.425418 \\
\hline LNFBKFRGOB & -22.15286 & -0.387304 & 1.672150 & -1.384713 & -0.425418 & 1.211125 \\
\hline
\end{tabular}

\section{I Lectura de los resultados econométricos}

El ejercicio realizado para la economía mexicana al ser de carácter doblelogarítmico permitió realizar un análisis de elasticidades que en términos generales confirman que el crecimiento económico en México obedece fundamentalmente a los factores trabajo y capital, aunque variables como el PIB y los acervos de capital rezagados no resultaron significativos.

En lo que respecta al papel que juegan las instituciones gubernamentales en términos de sus efectos físicos y sociales se demuestra que la inversión gubernamental en infraestructura productiva resultó altamente significativa aunque de signo contrario, mostrando que posiblemente la inversión gubernamental no se está ejerciendo de la forma adecuada ni en los sectores estratégicos para que puedan detonar el crecimiento económico. Por su parte al gasto social arrojó muy pobres resultados en materia de crecimiento económico durante el periodo, ya que el gasto público en salud resulta positivo pero poco significativo, situación que no sucede con el gasto público en educación que además de resultar poco significativo su coeficiente arroja un signo negativo; este último resultado puede leerse como que en México en materia educativa se gasta poco y mal.

Finalmente los resultados econométricos obtenidos muestran resultados interesantes ya que el ajuste medido por la R2 ajustado que es el coeficiente de determinación, es aceptable al $\mathbf{9 4 . 8 3 \%}$ para este tipo de estudios, la DW presentó mayores problemas al pasar las pruebas al $5 \%$ de significancia, por su parte la prueba de regresiones espurias fue pasada al incluir variables que cointegran en el largo plazo y no se tuvieron problemas de multicolinealidad, de heteroscedasticidad, de correcta especificación dinámica además de que las variables que resultaron estadísticamente significativas están en línea con lo estipulado por el enfoque teórico asumido. 


\section{Conclusiones}

El avance registrado en los últimos lustros por parte de los modelos de crecimiento económico ha permitido comprobar empíricamente la importancia de las instituciones públicas en materia de crecimiento económico por medio de un sinnúmero de investigaciones realizadas para las economías de los países avanzados como las de insuficiente desarrollo entre las que se encuentra la economía mexicana; en este sentido puede afirmarse que en el caso de la economía mexicana, la crisis económica y la adopción de una estrategia de desarrollo adverso a la intervención económica gubernamental, configuraron un escenario de bajo y errático comportamiento económico que no le permitió a las instituciones gubernamentales mexicanas seguir contribuyendo al crecimiento económico del país, como lo habían estado haciendo por más de cincuenta años en la etapa inmediata anterior conocida como estrategia sustitutiva de importaciones.

El abandono registrado en México en esta etapa en materia de desarrollo institucional fue de tal magnitud que cuando se le vincula con el crecimiento económico, su impacto es prácticamente insignificante cuando no contrario al mismo como se demuestra el ejercicio econométrico realizado en la presente investigación que incipientemente pretendió avanzar en el conocimiento de la vinculación existente entre ambos aspectos.

Los resultados preliminares de la investigación permiten afirmar que el aletargamiento y mayor fragilidad que caracterizan a la actual economía mexicana, se deben por una parte al desempeño deficiente de las instituciones públicas existentes en el país y al abandono y desaparición intencional de las mismas, producto de la adopción a nivel gubernamental y de política económica de una filosofía económica (neoliberalismo) que se manifiesta adversamente contraria a la intervención del Estado en la economía

Finalmente si se quiere recuperar la senda de crecimiento económico de más del $6 \%$ que por décadas caracterizó a la economía mexicana, lo primero que debe de hacerse, es reconocer el rol estratégico que las instituciones públicas pueden llegar a jugar en la recuperación de la dinámica económica, para posteriormente pasar a una etapa en donde se les provea de la atención política y presupuestaria necesaria, tal que se garantice su eficiencia y pleno funcionamiento vital no solo en términos de su aporte al crecimiento de la economía mexicana, sino también para la estabilidad política y social de México. 
Economía Informa núm. $379 \cdot$ marzo - abril $\cdot 2013= \pm \square= \pm \square \square \square$

\section{Bibliografía}

Acemoglu, Daron (2004), Understanding Institutions, en: Lionel Robins, Lectures, 2004, Reino Unido.

Altamirano, Aniel (2005), Crecimiento Económico, Teoría y evidencia empírica del enfoque neoclásico, Ed. Galilei, México pp. 356.

Arrow, Keneth (1962), The Economic Implications of Learning By Doing, en: Review Of Economic Studies, No. 29, pp. I55- 173.

Ayala, Espino (2002), Fundamentos institucionales del mercado, Ed. FE de la UNAM, México, Pp. 253.

Bandeira, Pablo (2009), Instituciones y desarrollo económico un marco conceptual, en: Revista de Economía Institucional,Vol. I I, No. 20,Venezuela, pp. 355-272.

Barro, Robert (1992), Determinant of Economic Growth in a Cross Section of Countries, Quarterly Journal of Economics, I06(2), pp. 407-443.

Barselay, Michael (2003), La nueva gestión pública: Un acercamiento a la investigación y el debate de las políticas, Ed. FCE, México, Pp.40I.

Basilio, Navarrete (2009), Evaluación de la gestión pública en México: el caso de la Secretaría de Desarrollo Social, Tesis de Licenciatura, México, pp. 190.

Birsch, Peter (2008), Introducción a la macroeconomía avanzada,Vol. I: crecimiento económico, Ed. McGraw-Hill, España, pp.4l0.

Blanchard, Oliver (2008), Macroeconomía, 4a. Edición, Ed. Pearson, México, pp. 703.

Campos, Nauro; Jefrey, Nugent (1998), Development performance, and the institutions of governance: evidence from East Asia and Latin America, California: University of Southern California, Department of Economic.

Congressional Budget Office (1993), Estimating the effects of NAFTA: an assessment of the economic models and other empirical studies.

Colander, David (1992), New Keynesian Economics in Perspective, Ed. Eastern Economic Journal, Fall, EUA. pp. 439-448.

(2004), Historia del Pensamiento Económico, Ed. CECSA, México, pp. 524.

(2006), Post Walrasian macroeconomics: beyond the dynamic stochastic general equilibrium model, Ed. Cambridge University Press, usA.

Díaz, Alejandro (2006), Un modelo de crecimiento económico, instituciones e integración económica e inversión extranjera directa de México con los Estados Unidos, en: Convergencia, UAEM, Vol. I 3 No. 4I, México, Págs. II7-139.

Díaz, Bautista; Sáenz, Enrique (2002), Productividad total factorial y el crecimiento económico de México, en: Economía y Desarrollo Vol. I No. I, Colombia.

Domar, Evsey (1974), Expansión y empleo en: Mueller, Martin Lecturas de Macroeconomía, España, pp. 290-308. 
Estrada, López (2000), Nuevos modelos de crecimiento endógeno en México, en: Revista Análisis Económico, segundo semestre, Vol. xv, no. 32, México, pp. 3-4I.

Fondo Monetario Internacional (20I I), Ranking de Economías Mundiales.

Hanusch, Horst; Pyka, Andreas (2007), The Principles of Neo-Schumpeterian Economics, en:

Cambridge Journal of Economics, no. 31, pp. 275-289.

Harrod, Royd (1974), La dinámica económica, en: Muelle Martin, Lecturas de Macroeconomía, España, pp. 309-321.

Lavoie, Marc (2005), La economía Postkeynesiana, Ed. Icaria, España, pp. I 20.

Levine, Ross; Renet, David (1992), A sensitivity Analysis of cross-country Growth Regressions, American Economic Review, 82(4), pp. 942-963.

Levine, Ross (1993), What We Have Learned About Policy and Growth from cross-country regressions, AEA papers and proceedings 83, pp. 426-430.

Loría, Eduardo (2007), Los acervos de capital de México. Una estimación, I 980.1 -2004.IV, en: El Trimestre Económico,Vol. LXXIv, no. 294, pp.475-485.

Lucas, Robert (2002), Lectures on Economic Growth, Ed. Harvard University Press, EUA. pp. 320.

Mankiw, Gregori (2006), Macroeconomía, Ed.Antoni Bosch, España, pp.826.

Manrique, Irma; Martínez, Ramón; Bautista, Jaime (1996), Crisis económica: ¿fin del intervencionismo estatal?, Ed. El caballito, México, pp. I53.

Martínez, Ifigenia (200I), Globalización, proyecto nacional y globalización democrática, en: Calderón, Jorge, México en un mundo global, Ed. Instituto de Estudios de la Revolución Democrática, México, pp. 308.

North, Douglas (1990), Instituciones, cambio institucional y desempeño económico, Ed. FCE, México, pp. 290.

Polanyi, Karl (1992), La Gran Transformación, Ed. Juan Pablos Editor, México, pp. 335.

Romer, Paul (1994), The Origins of Endogenous Growth, en: Journal of Economic Prospectivs, Vol. 8, no. I, EU., pp. 3-22.

(1986), Increasing Returns and Long-Run Growth, Journal of Political Economic, EU., no.94, pp. 1003-1037

Rogosinsky, Jacques (1997), La privatización en México. Razones e impacto, Ed. Trillas, México, pp. 255.

Ros, Jaime; Moreno, Juan (20 I0), Desarrollo y crecimiento en la economía mexicana. Una perspectiva histórica, Ed. FCE, México, pp.405.

Schumpeter, Joseph (1934), The Theory of Economic Development, Ed. Cambridge Massachussetts, Harvard University Press, EU., pp. 487.

Smith, Adam (2009), Una Investigación sobre la naturaleza y causas de la riqueza de las naciones, Ed.Tecnos, España. pp. 719. 
Economía Informa núm. 379 - marzo - abril - 2013 a

(2002), Fronteras de la Economía del Desarrollo. El futuro en perspectiva, Ed. Alfa Omega, Colombia, pp. 579.

Solow, Robert (1956), A Contribution to the Theory of Economic Growth, en: Quarterly Journal of Economic,Vol.70, no. I, pp. 65-94.

(1957), El cambio tecnológico y la función de producción agregada, en: Stiglitz, Joseph (1998) Más instrumentos y metas más amplias para el desarrollo. Hacia el Consenso PostWashington, en: CLAD Reforma y Democracia. no. 12, Venezuela, pP. 189-208. 